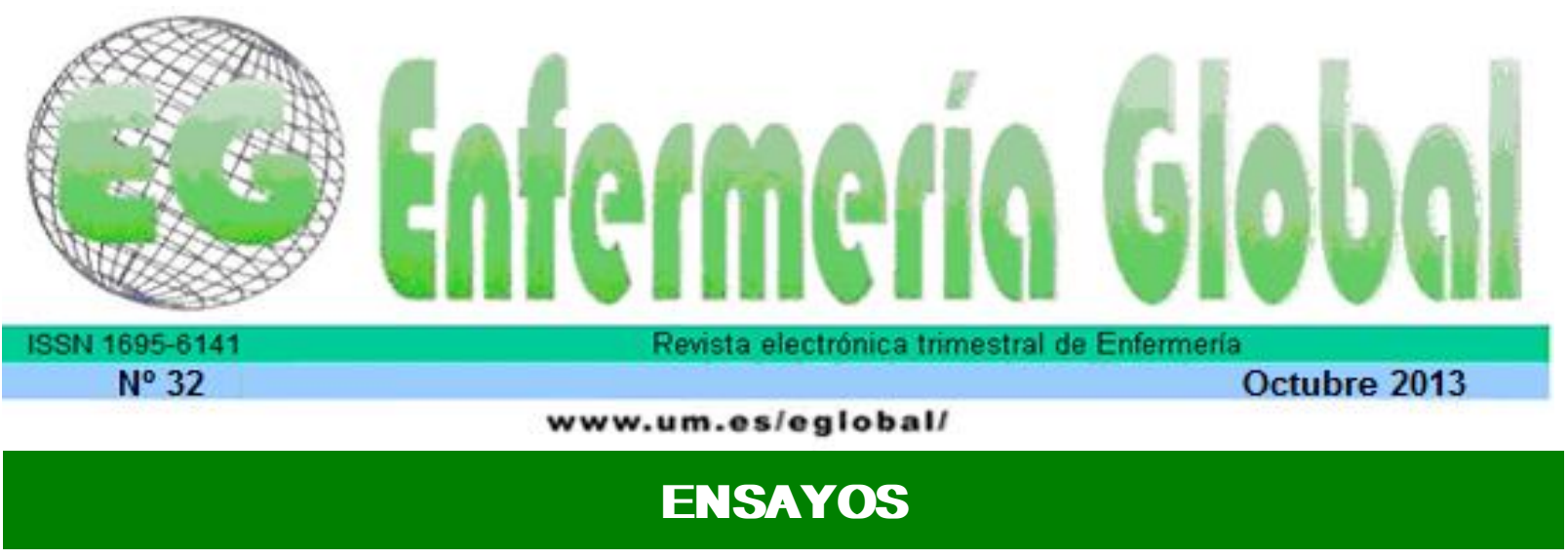

\title{
Promoción de la salud: la calidad de vida en las prácticas de enfermería
}

Promoção da saúde: a qualidade de vida nas práticas da enfermagem

Health promotion: the quality of life in nursing practices

\section{*Firmino Bezerra, Sara Taziana ** Mesquita Lemos, Aline *** Costa de Sousa, Sandra Maria ${ }^{* * * *}$ de Lima Carvalho, Carolina Maria ${ }^{* * * * *}$ Carvalho Fernandes, Ana Fátima ******Santos Alves, Maria Dalva}

*Universidade Federal do Ceará. Fortaleza-CE. Brasil. E-mail: saratfb@ yahoo.com.br

\author{
Palabras clave: Promoción de la salud; Calidad de vida; Enfermería; Cuidados de enfermería \\ Palavras chave: Promoção da saúde; Qualidade de vida; Enfermagem; Cuidados de enfermagem.
}

Keywords: Health promotion; Quality of life; Nursing. Nursing care.

\section{RESUMEN}

El objetivo fue reflexionar sobre la calidad de vida (CV) ofrecida en las prácticas de Enfermería con miras a la Promoción de la Salud, discutida desde la I Conferencia Internacional sobre Atención Primaria de Salud en Alma-Ata (1978), reforzada más tarde en las Conferencias sobre Promoción de la Salud y que tiene relación directa con la calidad de vida. Esta es entendida como la percepción de la persona sobre su vida, en el contexto cultural y social, asociada con los deseos, objetivos, expectativas e influenciada por el estado de salud. El enfermero desempeña papel importante en todos los niveles de atención, siendo profesional estratégico para proporcionar cambios en el estilo de vida de las personas que promueven la salud, por lo tanto, la calidad de vida, a través de su cuidado y del "empoderamiento" de la persona y su familia. Algunas experiencias confirman la intervención de enfermería con los pacientes con ciertas afecciones crónicas, individuales o colectivas, que promueven la salud. Es necesario sensibilizar cada vez más el personal de salud y de Enfermería, en particular, a promover la salud en el contexto del Sistema Único de Salud (SUS), para colaborar a la mejora de la calidad de vida de la sociedad.

\section{RESUMO}

Objetiva refletir acerca da qualidade de vida $(\mathrm{QV})$ propiciada nas práticas de Enfermagem com vistas à Promoção da Saúde, discutida desde a I Conferência Internacional sobre Cuidados Primários em Saúde, em Alma-Ata (1978), reforçada nas Conferências de Promoção da Saúde, posteriormente, e que tem relação direta com a qualidade de vida. Esta é entendida como a percepção da pessoa sobre sua vida, no contexto cultural e social, associado com os desejos, objetivos, expectativas e influenciada pelo estado de saúde. O enfermeiro exerce importante papel em todos os níveis de atenção, sendo 
profissional estratégico para proporcionar mudanças no estilo de vida das pessoas que promovam à saúde, consequentemente, a qualidade de vida, por meio do seu cuidado e do "empoderamento" da pessoa e de sua família. Algumas experiências confirmam a atuação da enfermagem junto a pacientes com certas condições crônicas, individuais ou coletivas, que promovem a saúde. Conclui-se ser necessário sensibilizar cada vez mais a equipe de saúde e a Enfermagem, em especial, no sentido de promover a saúde no contexto do Sistema Único de Saúde (SUS), que colaboram para a melhoria da qualidade de vida da sociedade.

\section{ABSTRACT}

This study aimed to reflect on the quality of life provided in nursing practices seeking Health Promotion, a discussion that happens since the International Conference on Primary Health Care in Alma-Ata (1978), later reinforced in the Conferences on Health Promotion and that is directly related to the quality of life. This is understood as the perception that someone has about their life in the cultural and social context, associated with desires, goals and expectations, and influenced by health conditions. The nurse plays an important role in all levels of care, being an essential professional to promote changes in the lifestyle of the people that promote health and consequently in the quality of life, through their care and "empowerment" of the person and their family. Some experiments confirm the health promotion of nursing practices with patients with certain chronic conditions, individual or collective. We conclude that it is necessary to increasingly sensitize the health and nursing staff, especially for health promotion in the context of SUS, who collaborate to improve the quality of life of society.

\section{INTRODUCCIÓN}

La Promoción de la Salud es discutida desde la I Conferencia Internacional sobre Cuidados Primarios en Salud, en Alma-Ata (1978), en Kazajistán, y fue reforzada em la I Conferencia sobre Promoción de la Salud, en Ottawa, Canadá (1986).

Inicialmente, se entiende la Promoción de la Salud como propuesta de "empoderamiento" de las personas, familias y comunidades, que permita su plena y efectiva participación en la discusión y elaboración de las políticas públicas, las cuales colaboran a la mejora de la calidad de vida..

El concepto se amplia con la idea de producción de ambientes saludables (sea el familiar, en el trabajo, en el ocio), buscando la reducción de las vulnerabilidades y, más recientemente, valorizando las redes sociales que fortalecen el soporte social.

En efecto, la Promoción de la Salud se relaciona con la calidad de vida en lo que concierne a los aspectos que influyen en las relaciones, la convivencia y el cotidiano en que se vive. La calidad de vida está asociada a factores objetivos (condiciones materiales necesarias para una supervivencia libre de miseria) y factores subjetivos (relaciones con otras personas, formación de identidades sociales, sentimiento de integración social y en armonía con la naturaleza). La calidad de vida aún está asociada a la capacidad (combinaciones de potencialidades y situaciones en que una persona es apta para ser o hacer) y a la funcionalidad (estados de una persona, las varias cosas que ella hace o es). La calidad de vida debe ser entendida por medio de las oportunidades efectivas de que las personas disponen para ser, realizaciones pasadas y presentes ${ }^{(1)}$.

Ante lo expuesto, esta reflexión tiene por objetivo dar a conocer la utilización por la Enfermería de la Promoción de la Salud, dentro del enfoque de la calidad de vida. Es sabido que el objeto de trabajo de la Enfermería es el cuidado al ser humano, lo que sucede en la interacción enfermero-cliente en situaciones tanto de enfermedad como de salud. 
Efectivamente, la cuestión es importante, en la medida en que busca pensar en la posición que la Enfermería asume en la política de Promoción de la Salud, su entendimiento por el enfermero, y cómo esto se reflejará en la calidad de vida de las personas.

En el sentido amplio de Promoción de la Salud, la Enfermería constituye campo de acción estratégico en el Sistema Único de Salud brasileño. El liderazgo para articular al equipo de salud, tanto en la atención primaria (auxiliares y técnicos de enfermería y agentes comunitarios de salud) como en la atención secundaria y terciaria (sin el segundo agente), permite al enfermero la posibilidad de valorizar las competencias individuales de esos miembros, para facilitar la interacción con el foco de cuidado, que es el paciente.

Se sabe, por eso, que ese modo de cuidar, aunque sea constantemente discutido en el ambiente de enseñanza- aprendizaje de futuros enfermeros, no implica que toda categoría trabaje con tales preocupaciones y atención. La integralidad del cuidado a la salud por cierto contribuirá a reducir el hiato que existe entre discurso y práctica por parte de la categoría de enfermeros, que dice ser el profesional que más tiempo permanece al lado del paciente/cliente, dándole cuidado integral y humanizado, aunque en la práctica, se involucre más con actividades administrativas gerenciales $^{(2: 24)}$.

El "empoderamiento" significa la posibilidad de la persona de asumir un mayor control sobre su propia vida, debiendo los profesionales de salud utilizar estrategias que buscan fortalecer la autoestima y la capacidad de adaptación al medio, desarrollando mecanismos de autoayuda y solidaridad ${ }^{(3)}$. Además de esto, la Promoción de la Salud abarca abordajes transdisciplinares, teniendo en cuenta la diferencia, la subjetividad y la singularidad de la vida, en la esfera individual y colectiva ${ }^{(4)}$. Estos son algunos de los determinantes sociales de la salud, que implican las condiciones de vida y de trabajo de las personas y grupos relacionados con su situación de salud ${ }^{(5)}$.

El interes por la temática surgió en el transcurso de la disciplina Enfermería y las Bases Teóricas de la Promoción de la Salud, del Programa de Post-Graduación en Enfermería de la Universidad Federal do Ceará. La disciplina propugna la necesidad de profundizar el conocimiento acerca del área de concentración - la Promoción de la Salud - con miras a la producción de la calidad de vida.

Se espera que esta reflexión estimule en los profesionales de salud la comprensión de la complejidad de la Promoción de la Salud, que exige la participación de los agentes por medio del "empoderamiento", privilegiando la calidad de vida, como objetivo a ser alcanzado, atendiendo a los diversos aspectos que influyen en este movimiento en la salud colectiva.

\section{USO DE LA PROMOCIÓN DE LA SALUD POR LA ENFERMERÍA}

El concepto de Promoción de la Salud surgió y se desarrolló en los últimos 20 años. Tres importantes conferencias internacionales establecieron bases conceptuales y políticas de Promoción de la Salud, las realizadas en Ottawa (1986), Adelaide (1988) y Sundsvall (1991). En ellas, el concepto de Promoción de la Salud todavía se 
confunde con el modelo tradicional de prevención de las enfermedades, acciones de inmunización, salud laboral y control de vectores.

La Promoción de la Salud está enfocada al ejercicio de la ciudadanía, participación de los sujetos con estrategia de transformaciones de las condiciones de vida, actividades que promuevan motivaciones, con planificación y evaluación de las efectividades de las acciones ${ }^{(6)}$. El conocimiento de Promoción de la Salude además de mejorar la escucha, fortalece los vínculos en que el modelo de intervención es participativo y en la movilización comunitaria.

En 1992, en el contexto de expansión y cualificación de la atención básica, se inició el primer programa. Se trata del Programa Agentes Comunitarios de Salud (PACS), siendo posteriormente transformado en política estructurada, conocido como Programa de Salud de la Familia, que se configuró como estrategia de reorientación del modelo asistencial, con el desafío de proporcionar mayor acceso a los servicios y participación comunitaria ${ }^{(6)}$.

Las concepciones del enfermero son fundamentales para su práctica, por ser quien detenta la competencia para actuar como educador, junto al equipo y la comunidad, y eso requiere un pensamiento con foco en los cambios de actitud. Las acciones del enfermero no pueden estar centradas en la prevención de enfermedades, en los factores de riesgo, cuyo objetivo está enfocado a la enfermedad.

Las acciones de Enfermería en Promoción de la Salud son fundamentales, con la realización de grupos para la conquista de la autonomía y asimilación de conocimientos en la mejora de la calidad de vida de las personas. Desarrollar acciones de cuidar pautadas en un proceso de diálogo, valorando la individualidad de cada ser, es un cuidado más próximo a las reales necesidades de los usuarios ${ }^{(7)}$.

Los conocimientos y las prácticas de Promoción de la Salud desarrollados en la formación del enfermero son necesarios para transformar las prácticas de enseñanza, superando el modelo biológico, y crear otro modelo operacional que supere las acciones preventivas, tanto en la formación, como en la actuación profesional. Es fundamental que el docente sea capaz de romper con prácticas tradicionales e implementar acciones a la luz de un nuevo paradigma ${ }^{(8)}$.

Se considera que el enfermero involucrado en la asistencia vivencia la oportunidad de aplicar estrategias de Promoción de la Salud, pues está en contacto directo con sus usuarios, participantes activos en los cuidados y mantenimiento de la salud. En este aspecto, la Promoción de la Salud se describe como el proceso de capacitación de la comunidad para actuar en la mejora de su calidad de vida y salud, incluyendo una mayor participación en el control de este proceso ${ }^{(3)}$. Acciones educativas son capaces de confirmar la autonomía ante situaciones de enfermedad. Usuarios de los servicios, familiares y profesionales están involucrados, estableciendo objetivos en común que puedan ser alcanzados.

Además, el enfermero interactúa con los otros agentes de la salud, a fin de facilitar la interdisciplinaridad, por medio de la cual los diversos saberes dialogan para proporcionar asistencias de calidad en los diversos contextos de cuidados.

Para la promoción de la salud en Brasil hay que considerar aspectos complejos como las desigualdades socioestructurales y las relaciones de poder injustas que afectan 
negativamente a más de un tercio de la población brasileña, aumentando el individualismo y la falta de solidaridad, perjudicando aún más la comunicación entre las personas. Esto deja imprecisa la definición de responsabilidades, imposibilitando cobros, si estas no son asumidas.

"Lo más preocupante es la gran distancia y una casi total imposibilidad de diálogo entre la elite de gestores y especialistas y la mayoría de la población en la base de la piramide social, usuaria de la salud pública, y para la que estas prácticas son supuestamente diseñadas" (9:234).

La interacción con el objeto de cuidado, en las situaciones de salud-enfermedad, se dirige a un encuentro entre sujetos, para satisfacer sus necesidades, sean fisiológicas, psicológicas o sociales. Este último campo, por ser más complejo, es un desafío al enfermero, mas este, de forma incipiente, demuestra el interés en articular los sectores que existen en el entorno de la persona, con miras a viabilizar la intersectorialidad, identificando potenciales referenciales que busquen la mejora de la calidad de vida de su comunidad.

Poco a poco, los enfermeros aprenden a pensar y a hacer Promoción de la Salud, un concepto amplio y complejo de ser incorporado en las personas, instituciones y sociedades, a fin de superar el tradicional patrón asistencial.

No es fácil despertar en el individuo, sin pensar en las posibles frustraciones, el interés en identificar aspiraciones, satisfacer necesidades y modificar favorablemente el medio ambiente ${ }^{(10)}$, ya que la propia Carta de Ottawa reconoció que el concepto positivo de salud y su promoción no es responsabilidad exclusiva del sector salud. Esta es una importante dimensión de vida, pero no la única.

En este sentido, la política de promoción de la salud incluye legislación, medidas fiscales, impuestos y cambios organizativos, lo que involucra gobierno, sector salud y otros segmentos sociales y económicos, organizaciones voluntarias y no gubernamentales, autoridades locales, industria y medios de comunicación ${ }^{(10)}$.

Hay una organización del concepto de salud, "a partir de la definición de la organización mundial de salud que la considera no como la ausencia de enfermedad, sino en una perspectiva positiva y ampliada, cuyo foco es el proceso social de su producción" (11).

Por ello, esta está conceptuada en la Política Nacional de Promoción de la Salud como

...una estrategia de articulación transversal en la que se da visibilidad a los factores que colocan la salud de la población en riesgo y las diferencias entre necesidades territoriales y culturales presentes en nuestro país, mirando a la variación de mecanismos que reduzcan las situaciones de vulnerabilidad, defiendan radicalmente la equidad e incorporen la participación y el control sociales en la gestión de las políticas públicas ${ }^{(12)}$.

El enfermero desarrolla sus acciones en los tres niveles de complejidad del SUS, y como tal, debe encajarse plenamente en la lucha por los valores por él preconizados, 
así como en la concretización del principio de la integralidad. Se trata de un discurso presente en la formación del enfermero de que el cuidado al paciente o cliente debe ser integral y de que sus acciones en tal actividad deben ser de totalidad, procurando cuidar siempre de modo holístico ${ }^{(2)}$.

El énfasis en la atención primaria en salud y las acciones específicas destinadas a la Promoción de la Salud exigen la movilización del enfermero de la Estrategia Salud de la Familia. El vínculo creado con las personas en su área de influencia lo hace referencia para la comunidad.

Esto facilita que su asistencia promueva la reflexión sobre la necesidad de alimentación saludable, fortaleciendo el estímulo al amamantamiento materno, la implantación de banco de leche, entre otros; en la escuela, incentivando la prática de actividades físicas en los propios espacios de la comunidad, incluyendo personas con deficiencias; sensibilizando a la comunidad para la prevención y control del tabaquismo; invirtiendo en la educación en salud de jóvenes y adultos sobre el uso abusivo de alcohol y drogas, responsables en gran parte de los índices de mortalidad, violencia, accidente de circulación; generando una cultura de paz y promoviendo en la comunidad el desarrollo sustentable ${ }^{(12)}$.

Los objetivos no son modestos ni fáciles de alcanzar, mas responden al objetivo de la estrategia "de reorientación del modelo asistencial, operacionalizada mediante la implantación de equipos multiprofesionales en unidades básicas de salud" (13).

Acciones de promoción y protección de la salud en las acciones de Enfermería tienden a ser relevantes en la promoción de la calidad de vida de sus clientes, por ello esta como derivada de la Promoción de la Salud, no es pertinente al foco de salud formal ${ }^{(13)}$.

\section{CONCEPTO DE CALIDAD DE VIDA}

La expresión calidad de vida (CV) es comentada desde muy antiguo por varios autores y constituye por excelencia uno de los conceptos más complejos para ser definido. La gran dificultad encontrada por investigadores en su definición es que la percepción de calidad de vida está unida a una subjetividad intrínseca, o sea, es la percepción de cada ser juntamente con determinadas condiciones, no privilegiando un modelo patronizado ${ }^{(14)}$.

La primera vez que la expresión CV fue mencionada data de 1920, por el autor Pigou, al escribir su libro en que se reporta la economía y bienestar, teniendo como foco el soporte que el gobierno realizaba para clases sociales con bajo nivel, repercusión en la vida de estas y el presupuesto del Estado ${ }^{(15)}$. Otros investigadores afirman que la palabra fue citada por primera vez y comenzó a destacar, después del año 1964, por el presidente Lyndon Johnson, de los Estados Unidos, cuando declaró que "los objetivos no pueden ser medidos a través del balance de los bancos. Ellos solo pueden ser medidos a través de la calidad de vida que proporcionan a las

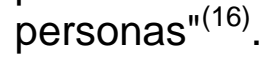

En 1995, un grupo de especialistas de la Organización Mundial de la Salud (OMS) en un trabajo multicéntrico, de culturas diferentes, definió CV como la percepción del individuo sobre su posición en la vida, en el contexto de la cultura y de los sistemas 
de valores en los cuales vive, y en relación a sus objetivos, expectativas, patrones y preocupaciones $^{(17)}$.

Muchos profesionales, incluso los del área de salud, ligan la expresión buena salud o bienestar con calidad de vida, asociando el significado de que tener salud es de hecho tener calidad de vida. Esto reduce la idea y restringe el modelo biomédico centrado en la enfermedad, en la ausencia de salud. Por tanto, como portador de enfermedad, no alcanzará la calidad de vida, mas ¿será posible tener calidad de vida siendo portador de una patología?

La calidad de vida ligada a la salud (CVLS) es definida como valor atribuido a la vida, ponderado por los deterioros funcionales; las percepciones y condiciones sociales que son inducidas por la enfermedad, agravamientos, tratamientos; y la organización política y económica del sistema asistencial ${ }^{(18)}$.

Hay tres vertientes para la expresión calidad de vida: calidad de vida (CV) como unidad de idea genérica de forma general, calidad de vida ligada a la salud (CVLS) y calidad de vida en el trabajo (CVT). ¿Puede limitarse a una sola definición y evaluar la calidad de vida en las colectividades o solamente en lo individual? ¿Cuál de las tres vertientes es la que trabaja mejor la calidad de vida? ¿O una complementa a la otra?

En una investigación realizada con estudiantes de Enfermería en la ciudad de São Paulo sobre el significado de calidad de vida y factores que la favorecen y la comprometen, se reveló que existen diferentes visiones de la expresión, y que en el transcurso de la formación, hay factores que favorecen y comprometen la calidad de vida, como, por ejemplo, el estrés. El estudio destaca el papel de la Universidad, no sólo en la enseñanza técnica, sino también en la concienciación sociocultural del enfermero en formación ${ }^{(19)}$.

\section{¿CÓMO LA ENFERMERÍA USA LA PROMOCIÓN DE LA SALUD CON ENFOQUE EN LA CALIDAD DE VIDA?}

La CV está incentivada en todos los niveles de atención, en que cada vez más son estudiadas las enfermedades crónicas y sus tratamientos, analizando el impacto de esas patologías en la calidad de vida de las personas.

El enfermero ejerce un importante papel en los cuidados relacionados con la CV en la Promoción de la Salud. El "empoderamiento" y la Educación en Salud permiten cambio de actitud y capacitación a las personas/ comunidad, soporte para adhesión a los tratamientos, motivación para identificar factores que interfieren en el tratamiento, como la práctica de ejercicio, dieta saludable y la participación activa, o sea, la salud depende de la calidad de vida.

El análisis reflexivo evidenció la escasez de trabajos que aborden la temática. Es preciso que en el periodo de formación académica, los futuros profesionales sean preparados, considerando indispensable que la educación transpase las consideraciones biológicas. Por tanto, la implementación de estrategias deben emerger basadas en las necesidades individuales comprometidas con la comunidad, con miras a la calidad del servicio en la calidad de vida.

Así, es necesario crear espacios, invertir para mejorar la calidad de vida, comprendiendo los sentimientos de las personas, para orientar conductas en el 
enfrentamiento de sus limitaciones, las modificaciones ocurridas en sus vidas, identificando las necesidades y las alteraciones en la CV.

Los profesionales precisan aprender a afrontar situaciones de salud y enfermedad, con abordajes centrados en las personas. El encuentro con ellas ayuda a adquirir conocimientos y facilita acciones para el cambio en el estilo de vida, importante influencia en la CV como paseos, estiramientos, ocio, terapia comunitaria y actividades conmemorativas.

Los resultados muestran la necesidad de invertir en cambios, procedentes de iniciativas como reflexiones sobre el papel del profesional. Es importante resaltar que el desarrollo y la implementación de estrategias de la Promoción de la Salud son insuficientes. El contacto paciente-enfermero es reducido por la sobrecarga de trabajo burocrático, necesitando de profesionales cualificados en número suficiente para atenderlos, objetivando mejorar la capacitación de personas en el autocuidado, orientadas a la mejora de la calidad de vida de la población.

En un estudio efectuado en una Unidad Básica de Salud de la Familia en la ciudad de São Paulo, acerca de las prácticas de Promoción de la Salud en la vivencia en grupos, se observó que se realizan prácticas educativas en la atención a la salud y actividades pedagógicas, mirando a la elaboración del conocimiento a partir de las informaciones vivenciadas. Los grupos realizados son de ancianos, hipertensos, diabéticos y gestantes, por medio de encuentros diarios, en que hay intercambio de experiencias entre los participantes, socialización, pintura, canto, paseo, danza, discusión de varios temas con contenido programático, ejercicio de relajación, repercusiones de las enfermedades, alimentación saludable, teatro y diversiones. El grupo es un espacio donde los profesionales tienen la oportunidad de constituir el "empoderamiento", capacitando a la persona y comunidad para establecer la Promoción de la Salud ${ }^{(20)}$.

Investigación realizada con el equipo de Salud de la Familia en los Municipios de Belo Horizonte y Contagem, sobre la práctica de grupo como acción de Promoción de la Salud en la Estrategia Salud de la Familia (ESF) reveló las dificultades vivenciadas en el proceso de capacitar a la persona para actuar en la mejora de su calidad de vida. Los grupos son elaborados centrados en patologías específicas y con acciones restringidas y tradicionales y poco innovadoras, utilizadas para la racionalización del trabajo, orientada para consultas colectivas. Las estrategias de Educación y Salud con énfasis en el modo tradicional, y la falta de planificación de acciones de Promoción de la Salud aumentan la distancia entre los profesionales y los usuarios. Cabe al profesional de salud la implantación de metodología y la conducción del grupo por el coordinador con orientaciones capaces de producir impacto sobre la salud y la calidad de vida ${ }^{(21)}$.

Otro estudio efectuado con enfermeros de la Estrategia Salud de la Familia en el Municipio de Sobral - Ceará - buscó saber sobre las prácticas de los enfermeros en el control de la hipertensión arterial. El estudio muestra que hay un esfuerzo para promover la salud de esas personas, en el cual los enfermeros realizan acciones de Promoción de la Salud, sea en consultas individuales o acción colectiva, con todo existen algunos momentos en que este esfuerzo queda comprometido, a causa del exceso de demanda y de la ausencia de espacio físico adecuado. Para el control de la hipertensión, no bastan solo medidas de orientación, siendo necesario desarrollar 
estrategias para el cambio de actitud y adhesión al tratamiento, disminuyendo los factores de riesgo por medio de hábitos alimentarios y estilo de vida saludable ${ }^{(22)}$.

\section{A MODO DE REFLEXIÓN}

Como se ha expresado en esta reflexión, existe actualmente la necesidad de repensar los conceptos que fundamentan la práctica profesional, entre ellos el de Promoción de la Salud. Esta debe ser vista como base, cuyo pensamiento es buscar el "empoderamiento" de las personas, sensibilizarlas para la busca constante de mejoras en la calidad de vida de la comunidad.

Es un cambio de paradigma que posibilitará la viabilización efectiva de los principios del SUS de integralidad del cuidado y de universalidad del acceso a los servicios, con articulación entre los diversos sectores en los entornos de la vida cotidiana de las personas, y valorizados los saberes, asociando el cuidado profesional con la cultura y las formas de enfrentar los problemas de salud.

Se nota que hay todavía una cultura de la práctica de la Enfermería situada en cuidados curativos, hospitalocéntrica, en el modelo biomédico, lo que aleja la fundamentación de esta pautada en acciones básicas de Promoción de la Salud ${ }^{(23)}$.

La Enfermería evoluciona en este sentido, pero todavía hay que buscar mucho, con miras a tornarse praxis insertada en los profesionales y no como iniciativas individuales, cuyo impacto no es visualizado por la sociedad.

\section{REFERENCIAS}

1 Westphal MF. O movimento cidades/municípios saudáveis: um compromisso com a qualidade de vida. Ciência e Saúde Coletiva, 2000; 5(1):39-51.

2 Antunes MJM, Guedes MVC. Integralidade nos processos assistenciais na atenção básica. IN: Integralidade da atenção no SUS e a sistematização da assistência de enfermagem. Garcia TR, Egry EY. Porto Alegre: Artmed, 2010.

3 Carvalho SR, Gestaldo D. Promoção à saúde e empoderamento: uma reflexão a partir das perspectivas crítico-social pós-estruturalista. Ciência \& Saúde Coletiva, 2008. 13 (sup 2): 2029-40

4 Backes MTS, Rosa LM, Fernandes GCM, Becker SG, Meirelles BHS, Santos SMA. Conceitos de saúde e doença ao longo da história sob o olhar epidemiológico e antropológico. Rev. Enferm UERJ, 2009; 17(1):111-7

5 Buss PM, Pellegrini Filho A. A saúde e seus determinantes sociais. Physis: Rev. Saúde Coletiva. 2007; 17(1):77-93.

6 Secretaria de Saúde (CE). Manual das equipes de saúde da família. $2^{\underline{a}}$ ed. Fortaleza: SESA; 2004.

7 Freitas CASL, Silva MJ, Vieira NFC, Ximenes LB, Brito MCC, Gubert FA. Evidências de ações de enfermagem em promoção da saúde para um envelhecimento ativo. Estud. Interdiscipl. Envelhec; Porto Alegre, 2010, 15(2):265-77.

8 Silva KL, Sena RR, Grillo MIC, Horta NC. Formação do enfermeiro: desafios para a promoção da saúde. Esc. Anna Nery Ver Enferm. 2010;14(2).

9 Travesso-Yepez MA. Dilemas na promoção da saúde no Brasil: reflexões em torno da política nacional. Interface (Botucatu) [on line]. 2007; 11(22):223:8.

10 Brasil. Ministério da Saúde. Secretaria de Políticas de Saúde. Projeto Promoção da Saúde. As cartas de promoção da saúde. Brasília: Ministério da Saúde, 2002. 
11 Arantes RC, Martins JLA, Lima MF, Rocha RM, Carvalho R, Villela WV. Processo saúde-doença e promoção da saúde: aspectos históricos e conceituais. Revista de APS. 2008;11(2):189-98.

12 Brasil. Ministério da Saúde. Secretaria de Vigilância em Saúde. Política nacional de promoção da saúde. Brasília: Ministério da Saúde, 2006. 60p. (Série B. Textos Básicos de Saúde).

13 Buss PM, Carvalho Al. Desenvolvimento da promoção da saúde no Brasil nos últimos vinte anos (1988-2008). Ciênc. Saúde Coletiva [on line]. 2009;9(3):745-9.

14 Fleck MPA. A avaliação de qualidade de vida: guia para profissionais da saúde. Porto Alegre: Artmed, 2008. 228p.

15 Wood-Dauphinee S. Assessing quality of life in clinical research: from where have come and where are we going? J Clin Epi 1999; 52(4): 355-63.

16 Fleck MPA, Fachel O, Louzada S, Xavier M, Chachamovich E, Vieira G et al. Desenvolvimento da versão em português do instrumento de avaliação de qualidade de vida da organização mundial da saúde (WHOQOL-100) 1999. Rev Bras Psiquiatr 1999;21:19-28.

17 The WHOQOL Group. The World Health Organization quality of life assessment (WHOQOL): position paper from the World Health Organization. Soc. Sci. Med. 1995;41:1403-10.

18 Auquier P, Simeoni MC, Mendizabal H. Approches théoriques et méthodologiques de la qualité de vie liée à la santé. Rev. Prevenir 1997;33:77-86.

19 Oliveira BM, Mininel VA, Felli VEA. Qualidade de vida de graduandos de enfermagem. Rev. Bras. Enferm. 2011; 64(1): 130-135.

20 Costa RC, Rodrigues CRF. Percepção dos Usuários Acerca das Práticas de Promoção da Saúde Vivenciadas em Grupos em uma Unidade Básica de Saúde da Família. REV. ASP, 2010;13(4):465-75.

21 Horta NS, Sena RR, Silva MEO, Tavares TS, Caldeira IM. A Prática de Grupos como Ação de Promoção da Saúde na Estratégia Saúde da Família. REV. ASP, 2009;12(3):293-331.

22 Ximenes Neto FR, Melo JR. Controle da Hipertensão Arterial na Atenção Primaria em Saúde uma Análise das Práticas do Enfermeiro. Rev Enfermería Global, 2005; 6.

23 Costa MS, Silva MJ. Tendências da temática qualidade de vida na produção científica de enfermagem. Rev Bras Enferm 2004;57(2):208-11.

ISSN 1695-6141

() COPYRIGHT Servicio de Publicaciones - Universidad de Murcia 